\title{
Metabolic rate predicts the lifespan of workers in the bumble bee Bombus impatiens
}

\author{
Evan P. $\mathrm{KelemeN}^{1}$, Nhi $\mathrm{CAO}^{2}$, Tuan $\mathrm{CAO}^{3}$, Goggy Davidowitz ${ }^{4}$, Anna Dornhaus ${ }^{1}$ \\ ${ }^{1}$ Department of Ecology and Evolutionary Biology, University of Arizona, 1041 E. Lowell St., P.O. Box 210088, Tucson, \\ AZ 85721, USA \\ ${ }^{2}$ Entomology and Insect Science Graduate Interdisciplinary Program, University of Arizona, Tucson, AZ 85721, USA \\ ${ }^{3}$ Department of Biology, University of North Carolina at Charlotte, Charlotte, NC 28223, USA \\ ${ }^{4}$ Department of Entomology, University of Arizona, Tucson, AZ 85721, USA
}

Received 12 July 2018 - Revised 19 November 2018 - Accepted 14 December 2018

\begin{abstract}
The rate of living theory posits that higher metabolic rates negatively affect lifespan. This relationship would influence trade-offs among life history traits associated with energy production and allocation. These tradeoffs may also apply within a species, resulting in differences among individuals in life history traits. In this study, we use the bumble bee Bombus impatiens to test for a relationship between metabolic rate and lifespan. We measured the resting metabolic rates of workers throughout their lives and noted their lifespans in the laboratory. Our results show that (1) resting metabolic rate inversely correlated with potential lifespan and (2) resting metabolic rate was not affected by age. These results suggest that within a species, individual differences in life-history trade-offs may exist as predicted by the rate of living theory.
\end{abstract}

metabolic rate / lifespan / aging / social insects / bumble bees

\section{INTRODUCTION}

Why do some individuals die sooner than others? One of the most debated theories for what affects lifespan and aging is the "rate of living" theory (Pearl 1928). This theory postulates that the faster an organism's metabolism, the faster it ages and the shorter its lifespan (Pearl 1928; Beckman and Ames 1998; Speakman 2005). Although there has been some support for this hypothesis, including early work finding a negative relationship between metabolic rate and lifespan across mammals

Electronic supplementary material The online version of this article (https://doi.org/10.1007/s13592-018-0630-y) contains supplementary material, which is available to authorized users.

Corresponding author: E. Kelemen, evankelemen@email.arizona.edu Manuscript editor: Klaus Hartfelder
(Rubner 1908; Kleiber 1961), and more recently across snakes species (Bronikowski and Vleck 2010), and within a beetle species (Arnqvist et al. 2017), support is not universal, including some studies finding no relationship within fly species (Hulbert et al. 2004a, b; Melvin et al. 2007) and even counter-evidence finding a positive relationship between metabolic rate and lifespan among birds (Møller 2008), and in some mammals including among mice (Miller et al. 2002; Speakman et al. 2004), bats (Austad and Fischer 1991), and marsupials (Austad and Fischer 1991). The rate of energy metabolism and the trade-offs associated with allocating energy to maintenance likely correspond with other life-history traits (Stearns 1992; Pontzer et al. 2014; Ton and Martin 2016; Bengston et al. 2017). The life history of an organism will influence how it budgets its finite energy among the competing interests of growth, reproduction, and self-maintenance. Subsequently, any variation among individuals in their energy costs of 
self-maintenance, estimated from their resting metabolic rate, may have consequences for fitness (Burton et al. 2011).

Body size is a major life history trait that is associated with resting metabolic rate. Although larger organisms have higher metabolic rates, they tend to have lower mass-specific metabolic rates and longer lifespans than smaller organisms (Brody 1945; Kleiber 1932; Speakman 2005)(Bronikowski and Vleck 2010). Thus, body size must be accounted for when studying the relationship between metabolism and lifespan.

In this study, we test the rate of living theory using a unique model system, workers of the bumble bee Bombus impatiens. As a social insect, bumble bees allowed us to naturally reduce confounding factors, such as reproduction and genetic variation that may affect metabolism. Bumble bee workers are largely nonreproductive and are all sisters from a singly mated queen. Despite their high genetic relatedness, workers display a large degree of continuous size variation within a colony, up to 10 -fold by mass (Goulson 2003). The size variation arises due to differential feeding during development (Couvillon and Dornhaus 2009; Kelemen and Dornhaus 2018) enabling us to compare how body mass relates metabolic rate and lifespan. Additionally, bumble bee colonies can be maintained easily under laboratory conditions thereby allowing workers to be tracked over their lifespan (an average of 2269 days depending on species; Goldblatt and Fell 1987; Smeets and Duchateau 2003; Karise et al. 2016) and eliminating effects due to extrinsic mortality factors (Ricklefs 2001).

Most studies on energy metabolism involve a single measurement assuming that the measured resting metabolic rate is indicative of the organism throughout ontogeny. However, the effects of age on metabolic rate have received little attention, leaving this assumption largely untested (Moe et al. 2009). Therefore, by repeatedly measuring resting metabolic rate in bumble bees, we answer (1) whether metabolic rate affects potential lifespan across individuals and (2) whether metabolic rate changes with age within individuals.

\section{METHODS}

We obtained three colonies of Bombus impatiens, containing singly mated queens, from Koppert Biological Systems (Romulus, MI, USA) in 2009 and were housed in plastic $(25 \mathrm{~cm} \times$ $18 \mathrm{~cm} \times 10 \mathrm{~cm})$ or wooden $(38 \mathrm{~cm} \times 23 \mathrm{~cm} \times$ $8 \mathrm{~cm})$ nest boxes covered with transparent Plexiglas. Each nest box was connected to a separate foraging arena. The colonies were provided with $50 \%$ sugar solution and pollen ad libitum daily. Colonies were kept under laboratory conditions (10:14 light:dark cycle; $25 \pm 1{ }^{\circ} \mathrm{C} ; \sim 25 \%$ humidity) at the University of Arizona in Tucson, AZ, USA. We checked colonies daily for newly emerged workers (callows). Upon emergence, we marked callows with unique numbered tags.

The resting metabolic rate of individual workers was quantified as $\mathrm{CO}_{2}$ production at 25 $\pm 1{ }^{\circ} \mathrm{C}$ and $\sim 25 \%$ humidity, in a dark room when at rest. We defined a resting bee as an immobile bee with all her legs touching the floor. We made measurements randomly between 10:00 and 19:00 to control for the effects of circadian rhythm. We checked each worker's resting metabolic rate by placing them in a respirometry chamber $(870 \mathrm{ml})$. An air pump (Rena Air 200-ES, Rena, Charlotte, NC, USA) circulated air from the chamber (at a flow rate of $1000 \mathrm{ml} \mathrm{min}^{-1}$ ) into a $\mathrm{CO}_{2}$ gas infrared analyzer (LI-820, LI-COR, Lincoln, NE, USA) and back into the chamber. We used LI-COR software $(820-500 \mathrm{~V}$ 1.0.0) to record $\mathrm{CO}_{2}$ concentration (in parts per million) at 1-s intervals for 10-15 min. Due to individuals displaying discontinuous gas exchange, we identified all cycles and calculated for each recording the average $\mathrm{CO}_{2}$ production $\left(\mu \mathrm{g} \mathrm{CO}_{2} \mathrm{~s}^{-1}\right.$ ) per cycle. We excluded recordings without a complete cycle. To account for mass, we weighed the bees immediately after respirometry measurements using a digital scale (Ohaus, USA) to the nearest $0.00001 \mathrm{~g}$.

We determined if resting metabolic rate correlates with lifespan by measuring the resting metabolic rate of $(N=31)$ newly emerged workers on the day of emergence from two different colonies. We calculated lifespan as the number of days bees were alive as adults. To determine if resting metabolic rate changes with age, we measured initial 
resting metabolic rate on the day of $(N=34)$ or within 3 weeks of $(N=11)$ emergence from three different colonies. We then measured the resting metabolic rate of individuals every $2-4$ days until the bee died. Only workers with at least three resting metabolic rate measurements were used for testing for an effect of age on resting metabolic rate.

Statistical analyses were conducted using $\mathrm{R}$ (R Development Core Team 2013), and linear mixed models were conducted using the package nlme (Pinheiro et al. 2017). Following Zuur et al. (2009), we verified the underlying assumptions of these linear mixed models by visually inspecting their residuals. The residuals from the models using the resting metabolic rate and body mass data did not follow the normal distribution, so we log transformed this data.

Data from the lifespan experiment were analyzed using a linear mixed model with colony as a random factor. Four models were fitted for each predictor variable: resting metabolic rate as a main effect, body mass as a main effect, both resting metabolic rate and body mass as main effects, and resting metabolic rate, body mass, and the interaction between resting metabolic rate and body mass as main effects. We controlled for heteroscedasticity by allowing for a greater residual variance with increasing metabolic rate using the weights function (varFixed) (Zuur et al. 2009). Data from the aging experiment were analyzed using a linear mixed model with individual and colony as random factors. Factors included age and body mass. Four models were fitted for each predictor variable: age as a main effect, body mass as a main effect, both age and body mass as main effects, and age, body mass, and the interaction between age and body mass as main effects. All models, as well as null models (no main effects), were fitted using the nlme package (Pinheiro et al. 2012). After fitting, the models were compared using Akaike information criterion corrected for small sample size (AICc) (Burnham and Anderson 2003). If two models differed by a $\triangle \mathrm{AICc}$ greater than two, the model with the lower AIC is considered to fit statistically better (Richards 2005).

\section{RESULTS}

Lifespan was best predicted by a model that contained the interaction between log resting metabolic rate at emergence and log body mass ( $\triangle \mathrm{AICc}$ to next model $>3$ in all comparisons; Table SI and Table SII) and is the one presented (Table I). The negative effect of the interaction between resting metabolic rate and body mass suggests that as body size increases, the relationship between resting metabolic rate and lifespan grows increasingly negative (Figure 1). This relationship exists even when the longest- and shortest-lived workers are removed from the analysis (Table SIII). The model had colony as a random factor, and lifespans differed between

Table I. The best-fit linear mixed model predicting the lifespan of bumble bee workers. The model included colony as a random factor.

\begin{tabular}{llllr}
\hline \multicolumn{1}{c}{ Parameter } & \multicolumn{1}{c}{$\beta^{1}$} & $\mathrm{SE}^{2}$ & $d f^{3}$ & $p$ \\
\hline Intercept & 59.492 & 15.374 & 29 & $<0.001$ \\
Log resting metabolic rate & 8.382 & 4.953 & 29 & 0.091 \\
Body mass & -197.267 & 102.57 & 29 & 0.054 \\
Log resting metabolic rate $\times$ body mass & -84.624 & 37.603 & 29 & 0.024 \\
& \multicolumn{3}{c}{ Marginal $R^{2}=0.131$} \\
\end{tabular}

\footnotetext{
${ }^{1}$ Effect size

${ }^{2}$ Standard error

${ }^{3}$ Degrees of freedom
} 


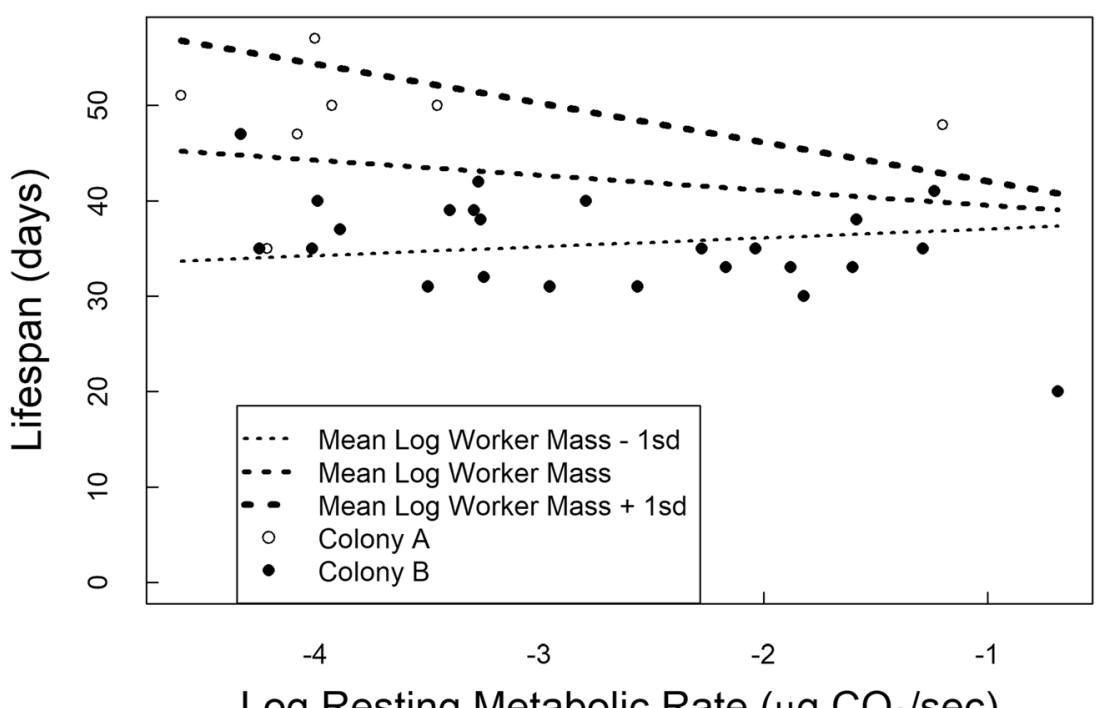

Figure 1. The relationship between worker lifespan and resting metabolic rate. Points represent individual workers $(N=31)$. The lines are the relationship between lifespan and log resting metabolic rate predicted by the best-fit model from Table I for workers with an average body mass and workers with body mass of mean +1 sd or mean 1 sd.

the colonies when analyzed separately (Figure S1). Average worker body mass and average metabolic rate did not differ between colonies (Figure S2, Figure S3).

Metabolic rate was best predicted by a model that contained log body mass but not age ( $\triangle \mathrm{AICc}$ to next model $>3$ in all comparisons; Table SIV and Table SV) and is the one presented (Table II). There was a significant positive relationship between log resting metabolic rate and log body

Table II. The best-fit linear mixed model predicting the (log) resting metabolic rate of bumble bee workers. The model included individual nested within colony as a random factor.

\begin{tabular}{llccc}
\hline \multicolumn{1}{c}{ Parameter } & $\beta^{1}$ & $\mathrm{SE}^{2}$ & $d f^{3}$ & $p$ \\
\hline Intercept & -1.566 & 0.419 & 234 & $<0.001$ \\
Log body mass & 0.949 & 0.199 & 234 & $<0.001$ \\
& \multicolumn{4}{c}{ Marginal $R^{2}=0.076$} \\
\\
\multicolumn{4}{c}{ Conditional $R^{2}=0.098$} \\
\hline
\end{tabular}

\footnotetext{
${ }^{1}$ Effect size

${ }^{2}$ Standard error

${ }^{3}$ Degrees of freedom
}

mass (Figure 2). This positive relationship appears isometric as the slope is greater than zero (slope $=$ $\left.0.94, t_{234}=4.765, p<0.001\right)$ but not significantly different from one $\left(t_{234}=0.258, p=0.375\right)$.

\section{DISCUSSION}

Overall, we found, in accordance with the rate of living theory, that bumble bee workers (B. impatiens) with higher resting metabolic rate at emergence have reduced lifespans when excluding external causes of mortality (such as predators). Metabolic rate explained $17.3 \%$ of lifespan variation (Table I). Interestingly, colony identity drove most of the variation, perhaps due to genetic differences or queen quality. We also show that resting metabolic rate does not change with age. There are three novel aspects of our study. First, using the bumble bee (B. impatiens) as our study organism allowed us to naturally reduce and account for effects that reproduction, genetic variation, and body size could have on metabolic rates. Second, our study design and results suggest that metabolic rate is at least one intrinsic factor that is correlated with lifespan. In general, the lifespan of an individual is affected by both extrinsic and 
intrinsic mortality factors ( $\mathrm{Li}$ and Anderson 2013). Because our study was conducted under laboratory conditions where there was no predation, extreme weather, or starvation, effects of extrinsic factors on the lifespan of bumble bee workers in our study were minimized (Ricklefs 2001), allowing us to better understand factors that could affect potential lifespan. Third, this study is one of the few that have investigated changes of resting metabolic rate over time using a longitudinal approach, where metabolic rates were repeatedly measured within individuals as they aged. Most studies of metabolic aging use single measurements, in which case, it is assumed that the measured metabolic rate remains constant throughout an organism's ontogeny, or are crosssectional in nature (Moe et al. 2009), where metabolic rates are measured from age cohorts. The longitudinal approach with repeated measures that we use in this study reduces variation among individuals of different cohorts.

The observed inverse relationship between metabolic rate and lifespan may be a consequence of the metabolic process itself. The free-radical/ oxidative stress theory of aging was proposed in the 1950s (Harman 1956; Beckman and Ames 1998), which suggest that the accumulation of reactive oxygen species (ROS) and other free radicals, which are the byproducts of metabolism, leads to cellular damage, enhanced aging, and a shorter lifespan. Support for the rate of living and radical/oxidative stress theories is, however, inconsistent, especially in intraspecific studies (Speakman 2005). For example, in a study by Speakman et al. (2004), there was a positive association between metabolic rate and lifespan in a cohort of mice. The mechanism for this positive correlation was that mice having higher metabolic rates also had significantly more uncoupling, which is the presence of proton leak pathways that reduce the potential of the inner mitochondrial membrane and, hence, reduce the movement of electrons across the membrane. The reduction of electron movement across the inner mitochondrial membrane thereby reduces harmful ROS production in the mitochondrial membranes and can consequently increase lifespan (Speakman et al. 2004). Other studies have reported no correlation between metabolic rate and lifespan (reviewed in Speakman 2005), such as in Drosophila (Hulbert et al. 2004a) and the blowfly Calliphora stygia (Hulbert et al. 2004b). The fact that there are inconsistent associations between metabolic rate and lifespan in the literature suggest that lifespan may be partly affected by metabolic rate (Orzack 2003), and it is possible that under certain conditions, it may play a larger role than other variables. Nevertheless, the results from our study provide

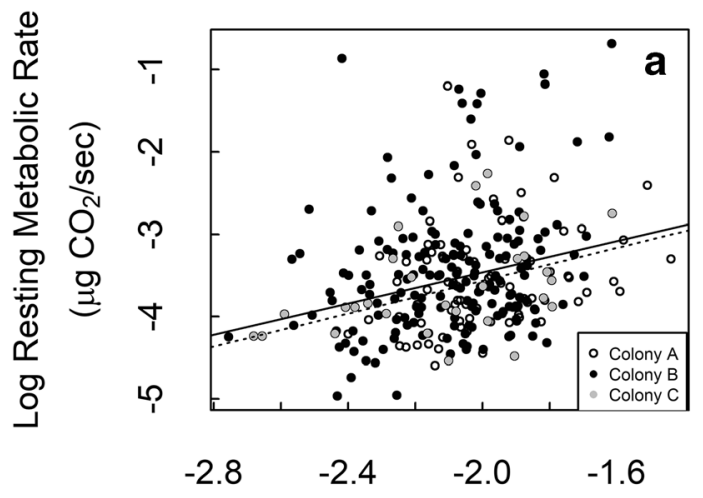

Log Body Mass (g)

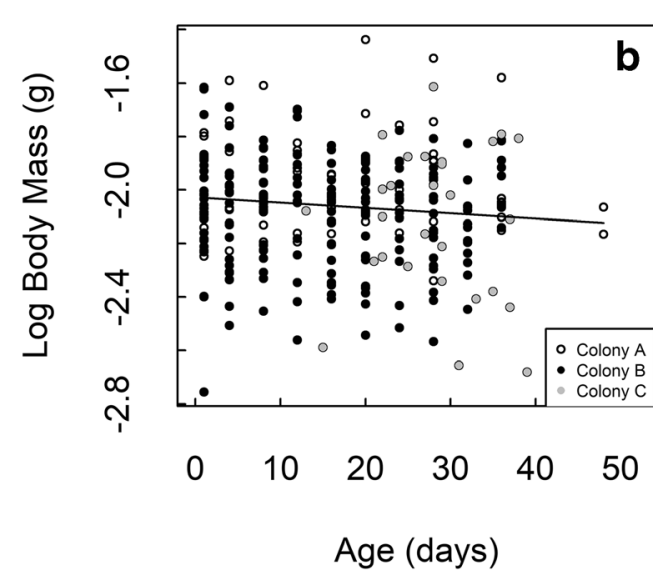

Figure 2 . The relationship between a resting metabolic rate and body mass and b body mass and age. Points represent measurements $(n=280)$ from 45 workers. a The solid line is calculated from Table II and has a slope of 0.95. The dashed line has a slope of 1 . b The line is calculated from a linear mixed effect model with age as a main effect and individual nested within colony as a random effect (effect size $=-0.002$, standard error $<0.001$, $p<0.001$, marginal $R^{2}=0.026$, and conditional $\left.R^{2}=0.562\right)$. 
support for the rate of living theory, and it is likely that our ability to detect this negative relationship is due to the fact that extrinsic factors of mortality were minimized in our laboratory setting, and by using sterile and highly related social insect workers, we were able to exclude the confounding effects of reproduction and reduce genetic variation.

It should be noted that for the free-radical/oxidative stress theory of aging, what is relevant is the cumulative metabolic rate required to meet an individual's daily energy expenditure. While, in this study, we only measured the resting metabolic rate, a relationship between resting metabolic rate and daily energy expenditure has been demonstrated (Daan et al. 1990; Hammond and Diamond 1997; Reinhold 1999), potentially from resting metabolic rate being physiological linked to maximum metabolic rate (Biro and Stamps 2010) or from resting metabolic rate being connected to daily activity levels (Biro and Stamps 2010). Bumble bee workers do vary in their activity level and type of tasks performed (Jandt et al. 2009), but this was not measured here.

Evolutionarily, resting metabolic rate and lifespan may be associated as part of an evolved "pace of life" syndrome. The idea of pace-of-lifesyndromes is inspired by the classic $\mathrm{r} / \mathrm{K}$ selection theory (Pianka 1970) and hypothesizes that populations and species under different environmental conditions should express different patterns of coevolved life-history traits including metabolism, physiology, reproduction, and behavior (Niemela et al. 2013). Individuals that grow fast, have a higher relative metabolism, shorter lifespan, and small body size are traditionally called "r-types". "K-type" individuals grow slowly, have a lower relative metabolism, longer lifespan, and larger body size. Within a bumble bee colony, different individuals may fall in different places along the $\mathrm{r} / \mathrm{K}$ continuum, and this could explain the negative relationship between resting metabolic rate and lifespan. However, it is unclear why a high resting metabolic rate is more detrimental to the lifespan of a large worker than to a small worker. This could be associated with the different tasks the bees are likely to do according to their size (Goulson et al. 2002; Jandt et al. 2009), as small workers are more likely to take care of the brood, and large workers are more likely to forage (Cumber 1949; Goulson et al. 2002). Foraging is energetically expensive. Previous work in honey bees (Schmid-Hempel and Wolf 1988) suggested a possible inverse relationship between workload and lifespan, and it has been demonstrated in house flies (Sohal and Buchan 1981) that there is a negative relationship between physical activity and lifespan. Increased physical activity may negatively affect lifespan through increased metabolic rates and rates of aging (Sohal and Buchan 1981). In honey bees, workers that initiate foraging at earlier ages die earlier (Rueppell et al. 2007, 2009), and reduction of mortality hazards or foraging efforts does not have strong effects of prolonging life which suggests that transition from non-foraging tasks to foraging itself has strong effects on honey bee worker lifespan (Rueppell et al. 2007). Within bumble bees, differences in resting metabolic rate may become amplified when foraging, therefore making small differences in metabolism more detrimental to large workers who forage. This hypothesis could be tested by comparing daily energy expenditure, which we would expect to show a negative relationship between lifespan and daily energy expenditure independent of an interaction with body size. We would also expect the difference between large and small workers to be larger among daily energy expenditures than resting metabolic rates due to the division of labor within the colony (Goulson et al. 2002; Jandt et al. 2009).

Our longitudinal approach to monitoring resting metabolic rate provides a more accurate description of whether energy metabolism changes with age because it eliminates biases due to disproportional mortality and inter-individual variation. Our result that resting metabolic rate does not change with age differs from previous studies in birds and humans (Roberts and Rosenberg 2006; Moe et al. 2009). Interestingly, the decrease in metabolic rate in previous studies may be attributed to a loss of lean body mass and organ mass (Even et al. 2001; Roberts and Rosenberg 2006). Here, bees did lose weight as they aged (Figure 2), but it did not affect their ability to maintain a constant absolute metabolic rate (Table II and Table SIII). This may be because the decrease in 
weight is due to water loss (Crailsheim 1985). There was considerable intra-individual variation in resting metabolic rate (up to a 28 -fold difference across measurements), which may result from circadian rhythms in metabolic rate (Southwick and Mortiz 1987), or task-dependent physiological changes that occur in an individual throughout its life (Elekonich and Roberts 2005). Despite the noise introduced by this variation, we found that metabolic rate increased isometrically with body size.

In summary, the negative correlation between resting metabolic rate and potential lifespan in bumble bee workers found in this study lends support to the rate of living theory. We also show that while bumble bee workers lost weight as they aged, they maintained a similar resting metabolic rate throughout their lives. Our results suggest that studies on social insects, where reproduction and genetic variation can be easily controlled for, may provide unique insights on how selection drives behavioral and physiological traits that directly affect the evolution of pace of life syndromes.

\section{ACKNOWLEDGEMENTS}

We thank Wulfila Gronenberg and anonymous reviewers for providing comments on earlier versions of this manuscript.

\section{AUTHOR CONTRIBUTIONS}

NC, TC, GD, and AD developed this study's concept and approach. NC and TC conducted the experiment. EPK, GD, and AD analyzed the data. EPK and NC wrote the manuscript. EPK, $\mathrm{GD}$, and $\mathrm{AD}$ participated in the revisions of the paper. All authors read and approved the finial manuscript.

\section{COMPLIANCE WITH ETHICAL STANDARDS}

Conflict of interest The authors declare that they have no conflict of interest.
Le taux métabolique prédit la durée de vie des ouvrières du bourdon Bombus impatiens

Taux métabolique / durée de vie / vieillissement / insectes sociaux / bourdons

\section{Metabolismusraten können die Lebensdauer von Arbeiterinnen der Hummel Bombus impatiens vorhersagen}

\section{Metabolismusraten / Lebensdauer / Alterung / soziale Insekten / Hummeln}

Publisher's Note Springer Nature remains neutral with regard to jurisdictional claims in published maps and institutional affiliations.

\section{REFERENCES}

Arnqvist G., Stojković, B., Rönn, J.L., Immonen, E.. (2017) The pace-of-life: a sex-specific link between metabolic rate and life history in bean beetles. Funct. Ecol.

Austad S.N., Fischer, K.E.. (1991) Mammalian aging, metabolism, and ecology: evidence from the bats and marsupials. J. Gerontol. 46 (2), B47-B53.

Beckman K.B., B.N. Ames. (1998) The free radical theory of aging matures. Physiol. Rev. 78, 547-581.

Bengston S.E., Shin, M., Dornhaus, A.. (2017) Life-history strategy and behavioral type: risk-tolerance reflects growth rate and energy allocation in ant colonies. Oikos 126 (4), 556-564.

Biro P.A., J.A. Stamps. (2010) Do consistent individual differences in metabolic rate promote consistent individual differences in behavior?. Trends Ecol. Evol. 25 (11), 653-659.

Brody, S. (1945) Bioenergetics and growth; with special reference to the efficiency complex in domestic animals. Reinhold, New York

Bronikowski A., D. Vleck. (2010) Metabolism, body size and life span: a case study in evolutionarily divergent populations of the garter snake (Thamnophis elegans). Integr. Comp. Biol. 50(5), 880-887.

Burnham K.P., Anderson, D.R.. (2003) Model selection and multimodel inference: a practical informationtheoretic approach. Springer Science \& Business Media, Berlin.

Burton T., Killen, S., Armstrong, J., Metcalfe, N.. (2011) What causes intraspecific variation in resting metabolic rate and what are its ecological consequences?. Proc. R. Soc. Lond. B Biol. Sci.

Couvillon M.J., A. Dornhaus. (2009) Location, location, location: larvae position inside the nest is correlated with adult body size in worker bumble-bees (Bombus 
impatiens). Proc. R. Soc. Lond. B Biol. Sci. 276 (1666), 2411-2418.

Crailsheim K. (1985) Distribution of haemolymph in the honeybee (Apis mellifica) in relation to season, age and temperature. J. Insect. Physiol. 31 (9), 707-713.

Cumber R. (1949) The biology of humble-bees, with special reference to the production of the worker caste. Trans. R. Entomol. Soc. Lond. 100 (1), 1-45.

Daan S., Masman, D., Groenewold, A.. (1990) Avian basal metabolic rates: their association with body composition and energy expenditure in nature. Am. J. Physiol.-Regul., Integr. Comp. Physiol. 259 (2), R333-R340.

Elekonich M.M., Roberts, S.P.. (2005) Honey bees as a model for understanding mechanisms of life history transitions. Comp. Biochem. Physiol. A Mol. Integr. Physiol. 141 (4), 362-371.

Even P.C., Rolland, V., Roseau, S., Bouthegourd, J.-C., Tomé, D.. (2001) Prediction of basal metabolism from organ size in the rat: relationship to strain, feeding, age, and obesity. Am. J. Physiol. Regul. Integr. Comp. Physiol. 28, 1887-1896.

Goldblatt J.W., Fell, R.D. (1987) Adult longevity of workers of the bumble bees Bombus fervidus (F.) and Bombus pennsylvanicus (De Geer)(Hymenoptera: Apidae). Can. J. Zool. 65 (10), 2349-2353.

Goulson D. (2003) Bumblebees: Behaviour and Ecology. Oxford University Press, New York.

Goulson D., Peat, J., Stout, J.C., Tucker, J., Darvill, B., et al. (2002) Can alloethism in workers of the bumblebee, Bombus terrestris, be explained in terms of foraging efficiency? Anim. Behav. 64 (1), 123-130.

Hammond K.A., Diamond, J.. (1997) Maximal sustained energy budgets in humans and animals. Nature 386 (6624), 457-462.

Harman D. (1956) Aging: a theory based on free radical and radiation biology. J. Gerontol. 11, 298-300.

Hulbert A.J., Clancy, D.J., Mair, W., Braekman, B.P., Gems, D., et al. (2004a) Metabolic rate is not reduced by dietary-restriction or by lowered insulin/IGF-1 signaling and is not correlated with individual lifespan in Drosophila melanogaster. Exp. Gerontol. 39 (8), 1137-1143.

Hulbert A.J., Usher, M.J., Wallman, J.F.. (2004b) Food consumption and individual lifespan of adults of the blowfly, Calliphora stygia: a test of the 'rate of living' theory of ageing. Exp. Gerontol. 39, 1485-1490.

Jandt J.M., E. Huang, A. Dornhaus. (2009) Weak specialization of workers inside a bumble bee (Bombus impatiens) nest. Behav. Ecol. Sociobiol. 63 (12), 18291836.

Karise R., Muljar, R., Smagghe, G., Kaart, T., Kuusik, A., et al. (2016) Sublethal effects of kaolin and the biopesticides Prestop-Mix and BotaniGard on metabolic rate, water loss and longevity in bumble bees (Bombus terrestris). J. Pest Sci. 89(1), 171-178.

Kelemen, E., Dornhaus, A.. (2018) Lower temperatures decrease worker size variation but do not affect fine- grained thermoregulation in bumble bees. Behav. Ecol. Sociobiol. 72 (10), 170.

Kleiber, M. (1932) Body size and metabolism. Hilgardia. 6 (11): 315-353

Kleiber, M. (1961) The fire of life. An introduction to animal energetics. The fire of life. An introduction to animal energetics.

Li, T., Anderson, J.J.. (2013) Shaping human mortality patterns through intrinsic and extrinsic vitality processes. Demogr. Res. 28(12), 341-372.

Melvin, R.G., Van Voorhies, W.A., Ballard, J.W.O.. (2007) Working harder to stay alive: Metabolic rate increases with age in Drosophila simulans but does not correlate with life span. J. Insect Physiol. 53, 1300-1306.

Miller, R.A., Harper, J.M., Galecki, A., Burke, D.T.. (2002) Big mice die young: early life body weight predicts longevity in genetically heterogeneous mice. Aging Cell 1 (1), 22-29.

Moe B., B. Rønning, S. Verhulst, C. Bech. (2009) Metabolic ageing in individual zebra finches. Biol. Lett. 5 : 86-89.

Møller A.P. (2008) Relative longevity and field metabolic rate in birds. J. Evol. Biol. 21 : 1379-1386.

Niemela P.T., N.J. Dingemanse, N. Alioravainen, A. Vainikka, R. Kortet. (2013) Personality pace-of-life hypothesis: testing genetic associations among personality and life history. Behav. Ecol. 24 (4): 935-941.

Orzack S.H. (2003) How and Why Do Aging and Life Span Evolve?, in: Carey J.R. and Tuljapurkar S. (Eds.), Life Span: Evolutionary, Ecological, and Demographic Perspectives., New York, The Population Council, Inc..

Pearl R. (1928) The Rate of Living. University of London Press, London.

Pianka E.R. (1970) On r- and K-Selection. Am. Nat. 104 (940): 592-597.

Pinheiro, J., Bates, D., DebRoy, S., Sarkar, D., R.C. Team. (2012) nlme: Linear and nonlinear mixed effects models. R package version 3: 103

Pinheiro, J., Bates, D., DebRoy, S., Sarkar, D., R.C. Team (2017) nlme: Linear and nonlinear mixed effects models (R package version 3.1-128, 2016). R software

Pontzer H., D.A. Raichlen, A.D. Gordon, K.K. SchroepferWalker, B. Hare, et al. (2014) Primate energy expenditure and life history. Proc. Natl. Acad. Sci. U.S.A. 111 (4): 1433-1437.

R Development Core Team (2013) A language and environment for statistical computing., R Foundation for Statistical Computing, Vienna.

Reinhold K. (1999) Energetically costly behaviour and the evolution of resting metabolic rate in insects. Funct. Ecol. 13 (2): 217-224.

Richards S.A. (2005) Testing ecological theory using the information-theoretic approach: examples and cautionary results. Ecology 86 (10): 2805-2814. 
Ricklefs R.E., Scheuerlein (2001) Comparison of agingrelated mortality among birds and mammals. Exp. Gerontol. 36 : 845-857.

Roberts S.B., I. Rosenberg. (2006) Nutrition and aging: changes in the regulation of energy metabolism with aging. Physiol. Rev. 86 : 651-667.

Rubner M. (1908) Das problem der Lebensdauer und seine Beziehungen sum Wachstum under Ehrnarung. Oldenburg: Munich.

Rueppell O., C. Bachelier, M.K. Fondrk, R.E.J. Page. (2007) Regulation of life history determines lifespan of worker honey bees (Apis mellifera L.). Exp. Gerontol. 42 (10): 1020-1032.

Rueppell O., O. Kaftanouglu, R.E.J. Page. (2009) Honey bee (Apis mellifera) workers live longer in small than in large colonies. Exp. Gerontol. 44 : 447-452.

Schmid-Hempel P., T. Wolf. (1988) Foraging effort and life span of workers in a social insect. J. Anim. Ecol. 57 (2): 509-521.

Smeets P., M.J. Duchateau. (2003) Longevity of Bombus terrestris workers (Hymenoptera: Apidae) in relation to pollen availability, in the absence of foraging. Apidologie 34 (4): 333-337.
Sohal R.S., P.B. Buchan. (1981) Relationship between physical activity and life span in the adult housefly, Musca domestica . Exp. Gerontol. 16 (2): 157-162.

Southwick E.E., R.F. Mortiz. (1987) Social synchronization of circadian rhythms of metabolism in honeybees (Apis mellifera). Physiol. Entomol. 12 (2): 209-212.

Speakman J.R. (2005) Body size, energy metabolism and lifespan. J. Exp. Biol. 208 : 1717-1730.

Speakman J.R., D.A. Talbot, C. Selman, S. Snart, J.S. McLaren, et al. (2004) Uncoupled and surviving: individual mice with high metabolism have greater mitochondrial uncoupling and live longer. Aging Cell 3 (3): 87-95.

Stearns, S.C. (1992) The evolution of life histories. Oxford University Press, Oxford.

Ton R., T.E. Martin. (2016) Metabolism correlates with variation in post-natal growth rate among songbirds at three latitudes. Funct. Ecol. 30 (5): 743-748.

Zuur, A., Ieno, E., Walker, N., Saveliev, A., Smith, G. (2009) Mixed effects models and extensions in ecology with R. Gail M, Krickeberg K, Samet JM, Tsiatis A, Wong W, editors. Spring Science and Business Media, New York 